\section{Evaluation of dry eye and meibomian gland dysfunction with meibography in vitiligo}

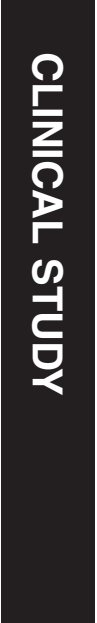

${ }^{1}$ Department of Ophthalmology, Ege University Faculty of Medicine, Izmir, Turkey

${ }^{2}$ Department of Dermatology, Ege University Faculty of Medicine, Izmir, Turkey

Correspondence: M Palamar, Department of Ophthalmology, Ege Universitesi Tip Fakultesi Hastanesi, Goz Hastaliklari AD, 35040 Izmir, Turkey Tel: +902323881469; Fax: +90 2323881469 . E-mail: melispalamar@ hotmail.com

Received: 3 August 2016 Accepted in revised form: 7 January 2017 Published online: 10 March 2017

This study is accepted as an oral presentation in XXXIV Congress of the ESCRS 2016, Copenhagen, Denmark.
M Palamar ${ }^{1}$, P Kiyat ${ }^{1}$, I Ertam ${ }^{2}$ and A Yagci ${ }^{1}$

Eye (2017) 31, 1074-1077; doi:10.1038/eye.2017.38;

published online 10 March 2017

\section{Introduction}

Vitiligo is a common chronic acquired disease of pigmentation. Although its etiology is unknown, it is characterized by destruction of melanocytes in the skin that causes hypopigmented and asymptomatic macules with sharply demarcated margins. ${ }^{1}$ A systemic destruction of melanocytes in various tissues-not only skin-especially in the mucous membranes, eyes, and the membranous labyrinth of the inner ear are also reported. An association of vitiligo, ocular manifestation, hearing loss, and autoimmune diseases have also been reported. ${ }^{2,3}$ Vitiligo affects both genders equally and can develop at any age. ${ }^{4}$ However, in $70-80 \%$ of the cases, it arises before the age of 30.4

The choroid and retinal pigment epithelium show similarities to the skin as they display the presence of well-differentiated melanocytes, with a common origin: the neural crest. In the eye, these melanocytes contribute to retinoid production and protection against ultraviolet rays. ${ }^{1}$ Hypopigmented spots on the iris, pigmentation on the anterior chamber, lenticular findings, chorioretinal degeneration, retinal pigment epithelial hypopigmentation, and uveitis are some of the ocular features detected in vitiligo patients. 6,7 Recently, a possible association of dry eye and vitiligo disease is also reported. 7,8

Meibomian glands are specialized sebaceous glands localized at the tarsal plates of the eyelids. They are responsible for lipid secretion that plays a significant role to build the ocular surface tension and prevent tear evaporation. ${ }^{9}$ Lipid layer abnormalities due to meibomian gland dysfunction (MGD) ends up with chronic 
Table 1 The demographics, dry eye tests, and meiboscores of the groups

\begin{tabular}{|c|c|c|c|}
\hline & Group 1 (Mean, SD, range) & Group 2 (Mean, SD, range) & P-value \\
\hline Age (years) & $35.5 \pm 13.4(8-54)$ & $35.2 \pm 12.4(8-52)$ & 0.942 \\
\hline Schirmer 1 (mm) & $15.7 \pm 7.8(0-30)$ & $19.4 \pm 7.7(10-35)$ & 0.139 \\
\hline T-BUT (s) & $12.5 \pm 5.8(3-27)$ & $16.4 \pm 6.2(7-32)$ & 0.047 \\
\hline Oxford scale & $0.4 \pm 0.6(0-2)$ & $0.1 \pm 0.3(0-1)$ & 0.057 \\
\hline OSDI score & $30.0 \pm 23.0(4.1-71.5)$ & $16.8 \pm 15.6(4.1-66.6)$ & 0.071 \\
\hline Upper meiboscore & $1.5 \pm 1.1(0-3)$ & $0.4 \pm 0.5(0-1)$ & 0.001 \\
\hline Lower meiboscore & $0.6 \pm 0.7(0-2)$ & $0.4 \pm 0.6(0-2)$ & 0.300 \\
\hline Total meiboscore & $1.1 \pm 0.6(0-2.5)$ & $0.4 \pm 0.5(0-1.5)$ & 0.003 \\
\hline
\end{tabular}

Abbreviations: OSDI, Ocular Surface Disease Index; T-BUT, tear film break-up time.

dry eye disease, which causes ocular irritation and ocular surface disorders. ${ }^{10}$ Recently, infrared imaging of meibomian glands and scoring systems for meibomian gland loss are popular research subjects on MGD. ${ }^{11,12}$

To our knowledge, no study to date has evaluated the meibomian glands of vitiligo patients with meibography. For this purpose, we evaluated dry eye and MGD with meibography in vitiligo patients and compared these results with healthy individuals.

\section{Materials and methods}

In this cross-sectional observational study, 20 right eyes of 20 vitiligo patients (Group 1) and 20 right eyes of 20 healthy volunteers (Group 2) were evaluated. All vitiligo patients included in the study were already diagnosed as vitiligo by a specialized dermatologist. Patients with any corneal problem (vascularization, scarring, and so on), cataract, glaucoma, contact lens use, previous ophthalmic surgery, and smoking history were excluded. Both eyes of each subject were evaluated for the study and the mean values of both eyes were assessed for data analysis.

Besides a detailed ophthalmological examination, corneal and conjunctival fluorescein staining (Oxford scoring), tear film break-up time (T-BUT; the mean time after three measurements (after each blink) was considered), Schirmer 1, and Ocular Surface Disease Index (OSDI) score assessment were performed to all subjects. Meibomian gland evaluation was performed by using infrared captures of a biomicroscope (Topcon, SL-D701) after everting both upper and lower eyelids. Partial or complete loss of the meibomian glands were scored for each eyelid as grade 0 (no loss of meibomian glands), grade 1 (the area characterized by gland dropout was $<1 / 3$ of the total meibomian glands), grade 2 (the area characterized by gland dropout was $1 / 3-2 / 3$ of the total meibomian glands), and grade 3 (the area characterized by gland dropout was $>2 / 3$ of the total meibomian glands). ${ }^{11}$ Meiboscore assessment was performed blinded by the same researcher (AY). The meiboscores for the upper and lower eyelids, and total (upper+lower) eyelids were summed for each eye.

Written informed consent was obtained from each subject. This study was approved by the institutional review board of the Ege University Hospital and adheres to the tenets of the Declaration of Helsinki. The Statistical Package for the Social Sciences version 11.5.0, Released 2002, SPSS for Windows (SPSS Inc., Chicago, IL, USA) was used for statistical analysis.

\section{Results}

The mean ages of vitiligo patients (Group 1) and healthy volunteers (Group 2) were $35.5 \pm 13.4$ (range, 8-54) and $35.2 \pm 12.4$ years (range, $8-52)$, respectively $(P=0.942$; Table 1). The mean BCVA of Group 1 and Group 2 were 1.00 (range, 1.00-1.00) and (range, 1.00-1.00), respectively $(P=1)$. Periocular involvement was detected in $40 \%$ of Group 1 patients.

Schirmer 1 measurements of Group 1 and Group 2 were $15.7 \pm 7.8$ (range, 0-30) and $19.4 \pm 7.7$ (range, 10-35) $\mathrm{mm}$, respectively $(P=0.139)$. T-BUT measurements of Group 1 and Group 2 were 12.5 55.8 (range, 3-27) and $16.4 \pm 6.2$ (range, $7-32$ ) s, respectively ( $P=0.047$ ). Dry eye was detected in seven patients (35\%) in Group 1 and none in Group 2 according to Schirmer 1 and T-BUT values. The mean superficial punctate staining according to Oxford scale and mean OSDI scores in Group 1 and Group 2 were statistically insignificant $(P=0.057$ and $P=0.071$, respectively).

The average upper eyelid, lower eyelid, and total (upper eyelid plus lower eyelid) eyelid meiboscores in Group 1 were $1.5 \pm 1.1$ (range, 0-3), $0.6 \pm 0.7$ (range, 0-2), and $1.1 \pm 0.6$ (range, $0-2.5$ ), respectively. The average upper eyelid, lower eyelid, and total eyelid meiboscores in Group 2 were $0.4 \pm 0.5$ (range, $0-1$ ), $0.4 \pm 0.6$ (range, $0-2$ ), and $0.4 \pm 0.5$ (range, $0-1.5$ ), respectively. The meiboscores for upper eyelids and total eyelids were significantly higher in Group $1(P=0.001$ and $P=0.003$, respectively). 
When vitiligo patients with and without periocular involvement were compared, Schirmer 1 and T-BUT values were $9.6 \pm 6.2$ (range, 0-20) and 19.7 \pm 6.1 (range, 10-30) $\mathrm{mm}$, and $8.0 \pm 4.7$ (range, $3-17$ ) and $15.5 \pm 4.5$ (range, $10-27) \mathrm{s}$, respectively $(P=0.003$ and $P=0.003)$. The mean Oxford scale and OSDI scores in patients with periocular involvement and those without were $0.8 \pm 0.7$ (range, 0-2) and $0.1 \pm 0.3$ (range, 0-1), $41.4 \pm 25.2$ (range, 2.1-71.5) and $22.3 \pm 18.8$ (range, 4.1-54), respectively $(P=0.011$ and $P=0.103)$.

The average upper eyelid, lower eyelid, and total eyelid meiboscores in vitiligo patients with periocular involvement were $1.8 \pm 1.3$ (range, $0-3$ ), $0.5 \pm 0.5$ (range, $0-1$ ), and $1.1 \pm 0.6$ (range, $0-2$ ), respectively. The average upper eyelid, lower eyelid, and total eyelid meiboscores in patients without periocular involvement were $1.3 \pm 0.9$ (range, 0-3), $0.7 \pm 0.8$ (range, $0-2$ ), and 1.0 \pm 0.7 (range, $0-2.5)$, respectively. Meiboscores of upper, lower, and total eyelids in the groups with periocular involvement and non-involvement did not differ statistically $(P=0.400$, $P=0.732$, and $P=0.684$, respectively).

\section{Discussion}

It is reported that about $1-2 \%$ of the world's population has vitiligo. ${ }^{5}$ Although the etiology of vitiligo is still unclear, autoimmunity is the leading suspected cause. Dry eye syndrome, also have an autoimmune inflammatory basis, and can be seen with both autoimmune and rheumatologic diseases. ${ }^{13,14}$ Hence, the coincidence of dry eye and vitiligo are very likely. In a recent study, vitiligo patients were found to have higher OSDI scores, shorter T-BUT, and higher corneal fluorescein staining positivity than in controls. ${ }^{8}$ They found the MGD and Schirmer test results to be similar. In another study that only evaluated the Schirmer test in terms of ocular surface evaluation found that Schirmer scores of vitiligo patients were lower than normal. ${ }^{7}$ Herein, we found that mean Schirmer 1 values were lower, and Oxford score, OSDI score, and meiboscores of lower eyelids were higher in vitiligo patients, however, in none of these parameters, the difference was statistically significant. T-BUT values were significantly lower, and meiboscores of upper eyelids and total (upper+lower) eyelids were significantly higher in vitiligo patients than in healthy individuals.

The facial involvement may be present up to $87 \%$ of generalized vitiligo patients with various severity. ${ }^{15}$ The periocular involvement is reported to be present in $60 \%$ of cases. In our study group of vitiligo patients which all have generalized form, $40 \%$ of patients had periocular involvement. It is shown that at perilesional zones of vitiligo areas, there is apoptosis of melanocytes and accumulation of $\mathrm{T}$ lymphocytes. ${ }^{16}$ It seems to be logical to predict more ocular surface consequences of vitiligo in patients with periocular involvement. Dogan et $a l^{8}$ reported that T-BUT and Schirmer test were shorter, and corneal fluorescein staining was more prominent in patients with periocular involvement that support the idea of the negative impact of periocular localization on the ocular surface. In this study group of vitiligo patients, mean Schirmer 1 and T-BUT values were lower, and mean OSDI and Oxford scale scores were higher in patients with periocular involvement than those without. Meiboscores of upper and total eyelids were higher in vitiligo patients with periocular involvement compared to those without, however, the difference was not statistically significant.

As a conclusion, vitiligo can be associated with dry eye and there are significant differences in meibomian gland morphology in patients with vitiligo when compared with those without. Although, more studies with larger group of patients are still needed, patients with vitiligoespecially those with periocular involvement-should be monitored for the presence of dry eye. In case of dry eye detection, the role of evaporative and aqueous components should be discriminated to promptly start appropriate treatment for maintenance of the ocular surface homeostasis.

\section{Summary}

What was known before

- Although, in earlier studies, dry eye was reported to associate vitiligo, the association of meibomian gland dysfunction as measured with meibography was not investigated.

What this study adds

- To the best of our knowledge, this is the first study to evaluate the meibography of vitiligo patients. Moreover, the results were interesting to support meibomian gland dysfunction in vitiligo patients.

\section{Conflict of interest}

The authors declare no conflict of interest.

\section{References}

1 Iannella G, Greco A, Didona D, Didona B, Granata G, Manno A et al. Vitiligo: pathogenesis, clinical variants and treatment approaches. Autoimmun Rev 2016; 15: 335-343.

2 Akay BN, Bozkir M, Anadolu Y, Gullu S. Epidemiology of vitiligo, associated autoimmune diseases and audiological abnormalities: Ankara study of 80 patients in Turkey. J Eur Acad Dermatol Venereol 2010; 24: 1144-1150.

3 Al-Mutairi N, Al-Sebeih KH. Late onset vitiligo and audiological abnormalities: is there any association? Indian J Dermatol Venereol Leprol 2011; 77: 571-576. 
4 Alikhan A, Felsten LM, Daly M, Petronic-Rosic V. Vitiligo: a comprehensive overview part I. Introduction, epidemiology, quality of life, diagnosis, differential diagnosis, associations, histopathology, etiology, and work-up. J Am Acad Dermatol 2011; 65: 473-491.

5 Ezzedine K, Lim HW, Suzuki T, Katayama I, Hamzavi I, Lan CC et al. Revised classification/nomenclature of vitiligo and related issues: the Vitiligo Global Issues Consensus Conference. Pigment Cell Melanoma Res 2012; 25: E1-13.

6 Biswas G, Barbhuiya JN, Biswas MC, Islam MN, Dutta S. Clinical pattern of ocular manifestations in vitiligo. J Indian Med Assoc 2003; 101: 478-480.

7 Karadag R, Esmer O, Karadag AS, Bilgili SG, Cakici O, Demircan YT et al. Evaluation of ocular findings in patients with vitiligo. Int J Dermatol 2016; 55: 351-355.

8 Dogan AS, Atacan D, Durmazlar SP, Acar M, Gurdal C. Evaluation of dry eye findings in patients with vitiligo. Pak J Med Sci 2015; 31: 587-591.

9 Knop N, Knop E. Meibomian glands. Part I. Anatomy, embryology and histology of the Meibomian glands. Ophthalmologe 2009; 106: 872-883.

10 Nelson JD, Shimazaki J, Benitez-del-Castillo JM, Craig JP, McCulley JP, Den S et al. The international workshop on meibomian gland dysfunction: report of the definition and classification subcommittee. Invest Ophthalmol Vis Sci 2011; 52: 1930-1937.

11 Arita R, Itoh K, Inoue K, Amano S. Noncontact infrared meibography to document age-related changes of the meibomian glands in a normal population. Ophthalmology 2008; 115: 911-915.

12 Palamar M, Degirmenci C, Ertam I, Yagci A. Evaluation of dry eye and meibomian gland dysfunction with meibography in patients with rosacea. Cornea 2015; 34: 497-499.

13 Nguyen CQ, Peck AB. Unraveling the pathophysiology of Sjogren syndrome-associated dry eye disease. Ocul Surf 2009; 7: 11-27.

14 Yagci A, Gurdal C. The role and treatment of inflammation in dry eye disease. Int Ophthalmol 2014; 34: 1291-1301.

15 Speeckaert R, van Geel N. Distribution patterns in generalized vitiligo. J Eur Acad Dermatol Venereol 2014; 28: 755-762.

16 Wu J, Zhou M, Wan Y, Xu A. CD8+ T cells from vitiligo perilesional margins induce autologous melanocyte apoptosis. Mol Med Rep 2013; 7: 237-241. 